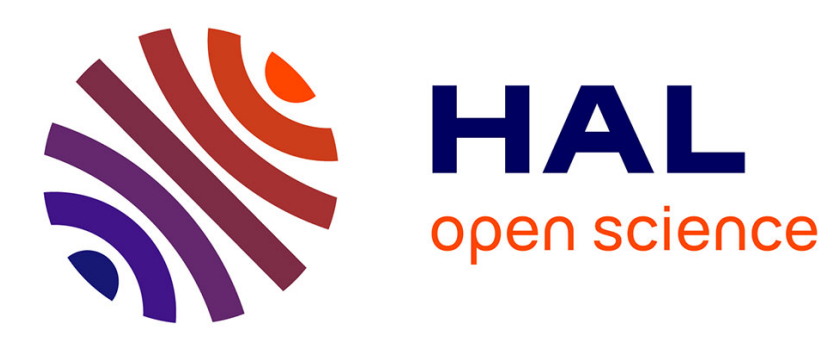

\title{
Theoretical Analysis of Flows Estimating Eigenfunctions of One-homogeneous Functionals
}

\author{
Jean-François Aujol, Guy Gilboa, Nicolas Papadakis
}

\section{To cite this version:}

Jean-François Aujol, Guy Gilboa, Nicolas Papadakis. Theoretical Analysis of Flows Estimating Eigenfunctions of One-homogeneous Functionals . SIAM Journal on Imaging Sciences, 2018, 11 (2), pp.14161440. hal-01563922

\section{HAL Id: hal-01563922 \\ https://hal.science/hal-01563922}

Submitted on 18 Jul 2017

HAL is a multi-disciplinary open access archive for the deposit and dissemination of scientific research documents, whether they are published or not. The documents may come from teaching and research institutions in France or abroad, or from public or private research centers.
L'archive ouverte pluridisciplinaire HAL, est destinée au dépôt et à la diffusion de documents scientifiques de niveau recherche, publiés ou non, émanant des établissements d'enseignement et de recherche français ou étrangers, des laboratoires publics ou privés. 


\title{
Theoretical Analysis of Flows Estimating Eigenfunctions of One-homogeneous Functionals for Segmentation and Clustering
}

\author{
Jean-Frangis Aujol $^{*} \quad$ Guy Gilboa ${ }^{\dagger} \quad$ Nicolas Papadakis ${ }^{\ddagger}$
}

July 18, 2017

\begin{abstract}
Nonlinear eigenfunctions, induced by subgradients of one-homogeneous functionals (such as the 1-Laplacian), have shown to be instrumental in segmentation, clustering and image decomposition. We present a class of flows for finding such eigenfunctions, generalizing a method recently suggested by Nossek-Gilboa. We analyze the flows on grids and graphs in the time-continuous and time-discrete settings. For a specific type of flow within this class, we prove convergence of the numerical iterations procedure and prove existence and uniqueness of the time-continuous case. Several examples are provided showing how such flows can be used on images and graphs.
\end{abstract}

\section{Introduction}

Eigenvalue analysis of linear operators is by now very well understood theoretically and has shown to be an essential framework for the analysis and understanding of many scientific and engineering problems. Consequently, a vast research was devoted to numerically solve eigenvalue problems [39, 24]. In recent years, there is a growing interest in nonlinear eigenvalue problems, which are based on nonlinear operators. Such problems appear in image processing $[9,26,34]$, computer vision [42], classification and learning $[13,30,28]$. In these problems the nonlinear operators are derived from norms, semi-norms or in general one-homogeneous functionals, where the

${ }^{*}$ IMB, Univ. Bordeaux, Jean-Francois.Aujol@math.u-bordeaux.fr

${ }^{\dagger}$ Technion - IIT, Haifa, Guy.Gilboa@ee.technion.ac.il

${ }^{\ddagger}$ CNRS, IMB, Nicolas.Papadakis@math.u-bordeaux.fr 
operator is essentially a subgradient element. In this paper we present a class of flows that converge to nonlinear eigenfunctions of one-homogeneous functionals.

We are interested in solving the following nonlinear eigenvalue problem

$$
\lambda u \in \partial J(u)
$$

where $J$ is a convex one-homogeneous functional, $\partial J(u)$ is the subdifferential and $\lambda$ denotes the eigenvalue. We refer to $u$ admitting (1) as an eigenfunction of $J$. More details and precise definitions are given in the following section.

A thorough investigation of such eigenfunctions was conducted for the case of the total-variation (TV) functional in the continuous setting. Meyer already observed in [33] that for the ROF problem [38] ( $T V-L^{2}$ square) for the case of a disk, the solution is the same disk with reduced contrast. In a series of studies $[1,2,7]$ shapes which preserve their shape under the TV gradient flow were characterized (termed calibrable sets). It was shown that convex characteristic sets in $\mathcal{R}^{2}$ with a certain bound on their curvature, are all eigenfunctions of TV. It was realized in a more general manner (see e.g. [19]) that eigenfunctions of one-homogeneous functional preserve their shape under three convex regularization methods - gradient flow, minimization with $L^{2}$ square and inverse-scale-space [20] (the time continuous form of Bregman iterations [37]). Thus one can view eigenfunctions essentially as atoms of the regularizer, having spatial features which are well preserved in the regularization procedure (up to some contrast change).

The above insights lead to attempts to decompose signals and images into distinct components based on eigenvalue analysis [9, 19, 40]. For the gradient flow with respect to one-homogeneous functionals, eigenfunctions decay linearly with respect to the time (flow) parameter and disappear at a finite time point. Thus taking the second time derivative of the solution of the flow yields a single response in time. This characteristic behavior was used to formulate a decomposition technique based on TV, referred to as spectral-TV decomposition [26], where certain nonlinear TV filters were defined in an analog manner to Fourier analysis. It was shown how one can extract desired features (and in particular eigenfunctions) in a range of scales (corresponding to eigenvalues) with high accuracy and with full contrast preservation. The method was later generalized to one-homogeneous functionals in [19] where certain properties, like orthogonality of the decomposed components, were shown in specific settings. Applications related to denoising [34], texture manipulation [29, 10] and segmentation of medical data [42] were suggested. 
In [9] nonlinear eigenfunctions for inverse problems (termed ground states) were investigated, the respective generalized Rayleigh quotient were analyzed and analytic examples of anisotropic TV were shown. Eigenfunctions related to the total-generalized-variation (TGV) functional [11] and to infimal convolution TV [23] were investigated in [35, 8] and properties of particular eigenfunctions of TGV were shown theoretically and numerically. Examples of certain eigenfunctions for different extensions of TV to color images were presented in [25].

In the field of machine learning it was shown $[14,18]$ that the Cheeger cut problem can be solved by solutions of the 1-Laplacian eigenvalue problem and consequently by minimizations of the total-variation functional on graphs. This was later developed in several studies for classification, clustering and segmentation in the binary- and multiple-class case $[12,13,30,28]$. Solutions of the Cheeger problem by using projections was shown in [21]. Uniqueness and regularity of Cheeger sets in $\mathbb{R}^{N}$ were analyzed in [22]. A flow, based on the MBO scheme [32], to refine graph-Laplacian eigenvectors for classification based on a diffuse interface model was proposed in [31]. An algorithm to construct particular TV eigenfunctions on graphs with certain regularity, referred to as nonlocal disks, was shown in [5].

In this work we present a family of new nonlinear flows, which considerably generalize the initial work of [36]. Moreover for a specific type of flow a comprehensive theoretical analysis is provided. Our proposed flows are very general, and can be evolved on both graphs and grids to solve various eigenvalue problems.

\section{$1.1 \quad$ Main contributions}

The main contributions of this paper are as follows:

1. We first analyze the flow of [36]. Then a generalized $\alpha$-flow is proposed for finding eigenfunctions. It is based on different normalizations between the function and its subgradient. A thorough analysis is presented along with a time discrete formulation of iterative convex optimizations to realize the flow.

2. For the specific case of $\alpha=1$ we are able to present a complete theory of the flow, including proof of convergence of the discrete case and existence and uniqueness of the time-continuous case.

The plan of the paper is the following. We first introduce some basic material for one homgeneous functionals in Section 2. We then analyse 
the flow of [36] in Section 3. We introduce a generalized $\alpha$-flow for finding eigenfunctions in Section 3.2. Section 4 is devoted to the particular case when $\alpha=1$ in the previous flow. For this specific choice of $\alpha$, we are able to prove existence and uniqueness of a solution, as well as the convergence to the solution of a numerical scheme. In Section 5, we illustrate our theoretical analysis with some numerical examples.

\section{One homogeneous functionals}

In this section, we outline some basic properties for one homogeneous functionals.

\section{$2.1 \quad$ Introduction}

We consider an absolutely one homogeneous functional $J$ that takes as input a function $u: x \in \Omega \rightarrow \mathbb{R}$ defined on a domain $\Omega \subset \mathbb{R}^{2}$. $\Omega$ can either be a discrete domain of size $|\Omega|=N$ or an open convex bounded set with Lipschitz boundary. $u$ are elements of some Hilbert space $X$ (e.g. $X$ can be $L^{2}(\Omega)$ ) embeded with some inner product $\langle.\rangle . J:, X \rightarrow \mathbb{R} \bigcup\{+\infty\}$ is assumed to be proper, convex and lower semi-continuous (lsc). Absolutely one-homogeneous functionals satisfy

$$
J(c u)=|c| J(u), \quad \forall c \in \mathbb{R}, \forall u \in X .
$$

The functional $J$ in finite dimensions can be, for instance, of the general form:

$$
J(u)=\sum_{i=1}^{N}\left(\sum_{j=1}^{N} w_{i j}\left|u_{i}-u_{j}\right|^{q}\right)^{1 / q}
$$

for $q \geq 1$, with $w_{i j} \geq 0$ (usually symmetric weights are assumed $w_{i j}=w_{j i}$ ). This formulation can be understood as a typical one-homogeneous functional on weighted graphs. In this case $u_{i}$ is the value of the function $u$ at node $i$ on the graph and $w_{i j}$ is the weight between node $i$ and node $j$. As grids of any dimension can be realized by specific graph structures, this formulation applies to standard grids as well. Thus (3), with appropriate weights, can be the spatial discrete version of anisotropic TV $(q=1)$, isotropic TV $(q=2)$ and anisotropic or isotropic nonlocal TV.

We recall the subgradient definition for general convex functionals

$$
p \in \partial J(u) \Leftrightarrow J(v)-J(u) \geq\langle p, v-u\rangle, \forall v .
$$


We also note the relation to the convex conjugate $J^{*}$

$$
J(u)=\sup _{p}\langle u, p\rangle-J^{*}(p)
$$

Below we state some properties of one-homogeneous functionals.

Property 1. A function $J$ defined in (3) admits:

(a) If $p \in \partial J(u)$, then $J(u)=\langle p, u\rangle$,

(b) If $p \in \partial J(u)$, then $J(v) \geq\langle p, v\rangle, \forall v$.

Notice in particular that from (b) we get that $\partial J(u) \subset \partial J(0) \forall u \in X$.

Property 2. The convex conjugate $J^{*}$ of a one-homogeneous functional is the characteristic function of the convex set $\{\partial J(0)\}$. Moreover, when $\Omega$ is included in a finite dimensional space, we have [19]:

$$
\exists C>0 \text { s.t. }\|p\|_{2} \leq C, \forall p \in \partial J(0) .
$$

From the equivalence of norms, we have that if $u$ is of zero mean, there exists a constant $\kappa>0$ for which

$$
\|u\|_{2} \leq \kappa J(u), \forall u \text { such that }\langle u, \mathbf{1}\rangle=0 .
$$

The nullspace of the functional is defined by

$$
\mathcal{N}(J)=\{u \in X \mid J(u)=0\} .
$$

The properties below are shown in [19].

Property 3. An absolutely one-homogeneous functional $J$ is a seminorm and its nullspace is a linear subspace.

Property 4. If a unit constant function $u=\mathbf{1}$ is in $\mathcal{N}(J)$ then any subgradient $p$ admits

$$
\langle p, \mathbf{1}\rangle=0 .
$$

We use $\ell_{2}$ and $\ell_{1}$ norms of $u$ defined as $\|u\|_{2}=\sqrt{\langle u, u\rangle}$ and $\|u\|_{1}=$ $\langle u, \operatorname{sign}(u)\rangle$. 


\subsection{Eigenfunctions of $J$}

In this work, we are interested in the eigenfunctions of functionals $J$ that are defined as follows.

Definition 1 (Eigenfunction of $J$ ). An eigenfunction of $J$ is a function that satisfies the eigenvalue problem (1), so that $J(u)=\langle\lambda u, u\rangle=\lambda\|u\|_{2}^{2}$ and $\lambda=\frac{J(u)}{\|u\|_{2}^{2}} \geq 0$.

An interesting insight on the eigenvalue $\lambda$ can be gained by the following proposition. We define $K=\{\partial J(0)\}$ to be the set of possible subgradients for any $u$. Indeed if $p \in \partial J(u)$ then $p \in \partial J(0)$. We first note that an eigenfunction that admits $\lambda u \in \partial J(u)$ has zero mean from Property 4 above. Next, as illustrated in Figure 1 we have the following result.

Proposition 1. For any non constant eigenfunction $u$, we have $\forall \mu \geq \lambda$,

$$
\lambda u=\operatorname{Proj}_{K}(\mu u),
$$

where $\operatorname{Proj}_{K}$ is the orthogonal projection onto $K=\{\partial J(0)\}$

Proof. If $u$ is a non constant eigenfunction, $\lambda u$ is on the boundary of $K$. As $K$ is bounded $\left(\|p\|_{2} \leq C, \forall p \in K\right)$ then for all $\mu>\lambda, \mu u \notin K$. Let us denote as $v$ the orthogonal projection of $\mu u$ onto $K$. For all $w \in K$ and $w \neq v, v$ satisfies:

$$
\begin{aligned}
\frac{1}{2}\|v-\mu u\|_{2}^{2} & <\frac{1}{2}\|w-\mu u\|_{2}^{2} \\
\frac{1}{2}\|v\|_{2}^{2}-2 \mu\langle v, u\rangle & <\frac{1}{2}\|w\|_{2}^{2}-\mu\langle w, u\rangle
\end{aligned}
$$

In particular, if we assume by contradiction that $v \neq \lambda u$ then:

$$
\begin{aligned}
\frac{1}{2}\|v\|_{2}^{2}-\mu\langle v, u\rangle & <\frac{1}{2}\|\lambda u\|_{2}^{2}-\mu\langle\lambda u, u\rangle \\
\frac{1}{2}\|v\|_{2}^{2}-\mu\langle v, u\rangle & <\frac{1}{2}\|\lambda u\|_{2}^{2}-\mu J(u) \\
\frac{1}{2}\|v\|_{2}^{2} & <\frac{1}{2}\|\lambda u\|_{2}^{2}
\end{aligned}
$$

since $J(u) \geq\langle v, u\rangle$. We thus have $\|v\|_{2}<\|\lambda u\|_{2}$ which yields $\frac{\|v\|_{2}}{\|u\|_{2}}<\lambda$. We denote $\tilde{v}=\frac{\|v\|_{2}}{\|u\|_{2}} u$ and observe that

$$
\|\mu u-\tilde{v}\|_{2}^{2}=\mu^{2}\|u\|_{2}^{2}+\|v\|_{2}^{2}-2 \mu\|u\|_{2}\|v\|_{2} \leq \mu^{2}\|u\|_{2}^{2}+\|v\|_{2}^{2}-2 \mu\langle u, v\rangle=\|\mu u-v\|_{2}^{2}
$$


and

$$
\|\mu u-\tilde{v}\|_{2}=\left(\mu-\frac{\|v\|_{2}}{\|u\|_{2}}\right)\|u\|_{2}>(\mu-\lambda)\|u\|_{2}=\|\mu u-\lambda u\|_{2}
$$

From (7) and (8) we get $\|\mu u-\lambda u\|_{2}<\|\mu u-v\|_{2}$ so $v$ can not be the orthogonal projection of $\mu u$ onto $K$.

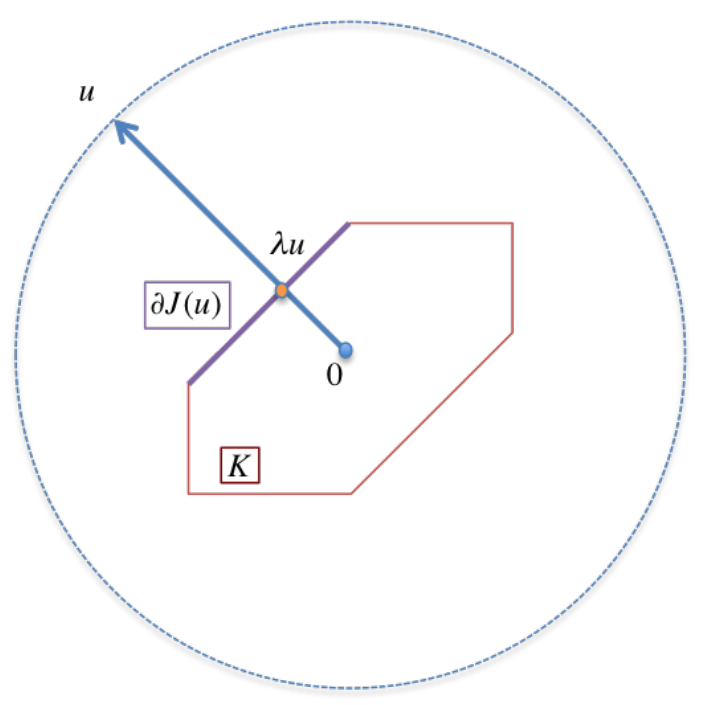

Figure 1: Illustration of an eigenfunction $u$ where $\lambda u \in \partial J(u) \subset K$. Observe that $\lambda u$ is the orthogonal projection of $u$ onto $K$.

\section{A flow for finding eigenfunctions of $J$}

In this section, following the method introduced in [36], we study flows for estimating eigenfunctions of one-homogeneous functions $J$ satifying Property 4.

\subsection{Introduction}

In order to find eigenfunctions of $J$, Nossek and Gilboa have introduced the flow [36]:

$$
\left\{\begin{array}{l}
u(0)=u_{0}, \\
u_{t}=\frac{u}{\|u\|_{2}}-\frac{p}{\|p\|_{2}}, \quad p \in \partial J(u) .
\end{array}\right.
$$


Proposition 2. Assume that there exists a solution $u$ of the flow (9). Then the following property holds:

$$
\frac{d}{d t} \frac{1}{2}\|u(t)\|_{2}^{2} \geq 0
$$

Moreover, we have:

$$
\|u(t)\|_{2} \leq\left\|u_{0}\right\|_{2}+t
$$

Proof. Recalling that $\langle p, u\rangle \leq\|p\|_{2}\|u\|_{2}$, this flow ensures that:

$$
\frac{d}{d t} \frac{1}{2}\|u(t)\|_{2}^{2}=\left\langle u, u_{t}\right\rangle=\left\langle u, \frac{u}{\|u\|_{2}}-\frac{p}{\|p\|_{2}}\right\rangle=\|u\|_{2}-\frac{\langle u, p\rangle}{\|p\|_{2}} \geq 0
$$

We can also remark that

$$
\frac{d}{d t} \frac{1}{2}\|u(t)\|_{2}^{2} \leq\|u(t)\|_{2}
$$

so that

$$
\|u(t)\|_{2} \leq\left\|u_{0}\right\|_{2}+t .
$$

Finally, if $u_{0}$ is of zero mean, Property 4 ensures that $u(t)$ is of zero mean, for all $t>0$.

Proposition 3. Assume that there exists a solution $u$ of the flow (9). Then the following property holds:

$$
\frac{d}{d t} J(u(t)) \leq 0 \text { for almost every } t .
$$

Moreover, $t \mapsto J(u(t))$ is non increasing for all $t \geq 0$.

Proof. We make use of Lemma 3.3 page 73 in [16] which states that $t \mapsto$ $J(u(t))$ is an absolutely continuous function (see also Lemma 4.1 in [3]). Moreover, recalling that $\langle p, u\rangle \leq\|p\|_{2}\|u\|_{2}$, this flow ensures that we have for almost every $t$ (using again Lemma 3.3 of [16]):

$$
\frac{d}{d t} J(u(t))=\left\langle p, u_{t}\right\rangle=\left\langle p, \frac{u}{\|u\|_{2}}-\frac{p}{\|p\|_{2}}\right\rangle=\frac{\langle u, p\rangle}{\|u\|_{2}}-\|p\|_{2} \leq 0 .
$$

This inequality holds for almost every $t$, and since $t \mapsto J(u(t))$ is an absolutely continuous function, we deduce that it is a non increasing function.

The PDE (9) converges iff $u_{t}=0$ so that

$$
p=\frac{\|p\|_{2}}{\|u\|_{2}} u \in \partial J(u) \Rightarrow p=\frac{J(u)}{\|u\|_{2}^{2}} u
$$

and $u$ is an eigenfunction of $J$ with eigenvalue $\lambda=\frac{J(u)}{\|u\|_{2}^{2}}$. 


\subsection{Generalized flow}

Let us now see the previous flow (9) as a specific instance of a more general framework. We define a flow for $\alpha \in[0 ; 1]$ as:

$$
\left\{\begin{array}{l}
u(0)=u_{0}, \\
u_{t}=\left(\frac{J(u)}{\|u\|_{2}^{2}}\right)^{\alpha} u-\left(\frac{J(u)}{\|p\|_{2}^{2}}\right)^{1-\alpha} p, \quad p \in \partial J(u) .
\end{array}\right.
$$

Notice that for $\alpha=1 / 2$, we retrieve the flow of Nossek and Gilboa (9), up to a normalization with $J^{1 / 2}(u)$.

Proposition 4. For $u_{0}$ of zero mean and $\forall \alpha \in[0 ; 1]$, the trajectory $u(t)$ of the PDE (13) satisfies the following properties:

(i) $\langle u(t), \mathbf{1}\rangle=0$.

(ii) $\frac{d}{d t} J(u(t)) \leq 0$ for almost every $t$. Moreover, $t \mapsto J(u(t))$ is non increasing. If $\alpha=0$, we have for almost every $t$ that $\frac{d}{d t} J(u(t))=0$ and $t \mapsto J(u(t))$ is constant.

(iii) $\frac{d}{d t}\|u(t)\|_{2} \geq 0$ and $\frac{d}{d t}\|u(t)\|_{2}=0$ for $\alpha=1$.

(iv) If the flow converge to $u^{*}$, we have $p^{*}=J^{2 \alpha-1}\left(u^{*}\right) \frac{\left\|p^{*}\right\|_{2}^{2(1-\alpha)}}{\left\|u^{*}\right\|_{2}^{2 \alpha}} u^{*} \in$ $\partial J\left(u^{*}\right)$ so that $u^{*}$ is an eigenfunction.

Proof. Property (iii) is obtained as follows:

$$
\begin{aligned}
\frac{d}{d t} \frac{1}{2}\|u(t)\|_{2}^{2} & =\left\langle u, u_{t}\right\rangle=\left\langle u,\left(\frac{J(u)}{\|u\|_{2}^{2}}\right)^{\alpha} u-\left(\frac{J(u)}{\|p\|_{2}^{2}}\right)^{1-\alpha} p\right\rangle \\
& =J^{\alpha}(u)\left(\|u\|_{2}^{2-2 \alpha}-\frac{J^{2-2 \alpha}(u)}{\|p\|_{2}^{2-2 \alpha}}\right) \geq 0 .
\end{aligned}
$$

For property (ii), we use once again Lemma 3.3 of [16]. For almost every $t$, it holds:

$$
\begin{aligned}
\frac{d}{d t} J(u(t)) & =\left\langle p, u_{t}\right\rangle=\left\langle p,\left(\frac{J(u)}{\|u\|_{2}^{2}}\right)^{\alpha} u-\left(\frac{J(u)}{\|p\|_{2}^{2}}\right)^{1-\alpha} p\right\rangle \\
& =J^{1-\alpha}(u)\left(\frac{J^{2 \alpha}(u)}{\|u\|_{2}^{2 \alpha}}-\|p\|_{2}^{2 \alpha}\right) \leq 0
\end{aligned}
$$

Since $t \mapsto J(u(t))$ is absolutely continuous (thanks to Lemma 3.3 of [16]), we deduce that it is non increasing. 


\subsection{Properties of a semi-explicit scheme}

We can look at the following semi implicit numerical scheme:

$$
\frac{u_{k+1}-u_{k}}{\delta t}=\left(\frac{J\left(u_{k}\right)}{\left\|u_{k}\right\|_{2}^{2}}\right)^{\alpha} u_{k+1}-\left(\frac{J\left(u_{k}\right)}{\left\|p_{k}\right\|_{2}^{2}}\right)^{1-\alpha} p_{k+1}
$$

It is easier to analyse this scheme than the previous continuous equation. Moreover, the properties that we prove on this scheme will be usefull in the next section.

Proposition 5. For $u_{0}$ of zero mean and $\delta$ such that $\frac{1}{\delta t}>\left(\frac{J\left(u_{k}\right)}{\left\|u_{k}\right\|_{2}^{2}}\right)^{\alpha}$, then the sequence $\left(u_{k}\right)$ is defined for all $k \geq 0$, and the trajectory $u_{k}$ given by the numerical scheme (14) satisfies:

$$
\begin{aligned}
& 1\left\langle u_{k}, \mathbf{1}\right\rangle=0 . \\
& \text { 2 } \frac{J\left(u_{k+1}\right)}{\left\|u_{k+1}\right\|_{2}} \leq \frac{J\left(u_{k}\right)}{\left\|u_{k}\right\|_{2}}, \\
& 3\left\|u_{k+1}\right\|_{2}^{2} \geq\left\langle u_{k+1}, u_{k}\right\rangle \geq\left\|u_{k}\right\|_{2}^{2} \\
& 4 \forall p_{k} \in \partial J\left(u_{k}\right),\left\|p_{k+1}\right\|_{2}^{2} \leq\left\langle p_{k+1}, p_{k}\right\rangle \leq\left\|p_{k}\right\|_{2}^{2} \text { and }\left\langle p_{k+1}, u_{k}\right\rangle \geq 0
\end{aligned}
$$

Proof. Let us rewrite the scheme (14) as

$$
\frac{u_{k+1}-u_{k}}{\delta t}=\beta_{k} u_{k+1}-\gamma_{k} p_{k+1}
$$

where $\beta_{k}=\left(\frac{J\left(u_{k}\right)}{\left\|u_{k}\right\|_{2}^{2}}\right)^{\alpha}$ and $\gamma_{k}=\left(\frac{J\left(u_{k}\right)}{\left\|p_{k}\right\|_{2}^{2}}\right)^{1-\alpha}$ for the sake of clarity. We define

$$
F\left(u, u_{k}\right)=\frac{1}{2 \gamma_{k} \delta t}\left\|u-u_{k}\right\|_{2}^{2}-\frac{\beta_{k}}{2 \gamma_{k}}\|u\|_{2}^{2}+J(u),
$$

$u_{k+1}$ is the unique minimizer of $F\left(., u_{k}\right)$, as soon as $\frac{1}{\delta t}>\beta_{k}$, i.e. $1-\delta t \beta_{k}>0$.

1 Let us underline that if $u_{0}$ is of zero mean, since $p$ is always of zeros mean, then $u_{k}$ is also of zero mean, so that property (i) of Proposition 4 is satisfied numerically.

2 If $\left\|u_{k}\right\|_{2}=0$, then $u_{k+1}=u_{k}=0$. Otherwise if $\left\|u_{k}\right\|_{2}>0$, we have: $F\left(u_{k+1}, u_{k}\right) \leq F\left(\frac{\left\|u_{k+1}\right\|_{2}}{\left\|u_{k}\right\|_{2}} u_{k}, u_{k}\right)$. Hence:

$$
\begin{aligned}
\frac{\left\|u_{k+1}-u_{k}\right\|_{2}^{2}}{2 \gamma_{k} \delta t}-\frac{\beta_{k}}{2 \gamma_{k}}\left\|u_{k+1}\right\|_{2}^{2}+J\left(u_{k+1}\right) & \leq \frac{\left\|\frac{\left\|u_{k+1}\right\|_{2}}{\left\|u_{k}\right\|_{2}} u_{k}-u_{k}\right\|^{2}}{2 \gamma_{k} \delta t}-\frac{\beta_{k}}{2 \gamma_{k}}\left\|u_{k+1}\right\|_{2}^{2}+\frac{\left\|u_{k+1}\right\|_{2}}{\left\|u_{k}\right\|_{2}} J\left(u_{k}\right) \\
\frac{1}{2 \gamma_{k} \delta t}\left\|u_{k+1}-u_{k}\right\|_{2}^{2}+J\left(u_{k+1}\right) & \leq \frac{1}{2 \gamma_{k} \delta t}\left(\left\|u_{k+1}\right\|_{2}-\left\|u_{k}\right\|_{2}\right)^{2}+\frac{\left\|u_{k+1}\right\|_{2}}{\left\|u_{k}\right\|_{2}} J\left(u_{k}\right) .
\end{aligned}
$$


As $\left\|u_{k+1}-u_{k}\right\|_{2}^{2}=\left\|u_{k+1}\right\|_{2}^{2}+\left\|u_{k}\right\|_{2}^{2}-2\left\langle u_{k}, u_{k+1}\right\rangle \geq\left\|u_{k+1}\right\|_{2}^{2}+\left\|u_{k}\right\|_{2}^{2}-$ $2\left\|u_{k+1}\right\|_{2} \cdot\left\|u_{k}\right\|_{2}=\left(\left\|u_{k+1}\right\|_{2}-\left\|u_{k}\right\|_{2}\right)^{2}$ then we deduce that

$$
J\left(u_{k+1}\right) \leq \frac{\left\|u_{k+1}\right\|_{2}}{\left\|u_{k}\right\|_{2}} J\left(u_{k}\right)
$$

3 We assume that $1-\delta t \beta_{k}>0$. First notice that from (15):

$$
\begin{aligned}
u_{k+1}\left(1-\delta t \beta_{k}\right) & =u_{k}-\gamma_{k} p_{k+1} \\
u_{k+1} & =\frac{1}{1-\delta t \beta_{k}}\left(u_{k}-\gamma_{k} \delta t p_{k+1}\right)
\end{aligned}
$$

Next, as $u_{k+1}=u_{k}+\delta t\left(\beta_{k} u_{k+1}-\gamma_{k} \delta t p_{k+1}\right)$, then:

$$
\begin{aligned}
\left\|u_{k+1}\right\|_{2}^{2} & =\left\|u_{k}\right\|_{2}^{2}+2 \delta t\left\langle u_{k}, \beta_{k} u_{k+1}-\gamma_{k} p_{k+1}\right\rangle+(\delta t)^{2}\left\|\beta_{k} u_{k+1}-\gamma_{k} p_{k+1}\right\|_{2}^{2} \\
& \geq\left\|u_{k}\right\|_{2}^{2}+2 \delta t\left\langle u_{k}, \frac{\beta_{k}}{1-\delta t \beta_{k}}\left(u_{k}-\gamma_{k} \delta t p_{k+1}\right)-\gamma_{k} p_{k+1}\right\rangle \\
& \geq\left\|u_{k}\right\|_{2}^{2}+2 \delta t\left(\frac{\beta_{k}}{1-\delta t \beta_{k}}\left\|u_{k}\right\|_{2}^{2}-\gamma_{k}\left(\frac{\beta_{k} \delta t}{1-\delta t \beta_{k}}+1\right)\left\langle u_{k}, p_{k+1}\right\rangle\right) \\
& \geq\left\|u_{k}\right\|_{2}^{2}+\frac{2 \delta t}{1-\delta t \beta_{k}}\left(\beta_{k}\left\|u_{k}\right\|_{2}^{2}-\gamma_{k} J\left(u_{k}\right)\right) .
\end{aligned}
$$

We now recall that $\beta_{k}=\left(\frac{J\left(u_{k}\right)}{\left\|u_{k}\right\|_{2}^{2}}\right)^{\alpha}$ and $\gamma_{k}=\left(\frac{J\left(u_{k}\right)}{\left\|p_{k}\right\|_{2}^{2}}\right)^{1-\alpha}$, hence:

$$
\begin{aligned}
\beta_{k}\left\|u_{k}\right\|_{2}^{2}-\gamma_{k} J\left(u_{k}\right) & =\left(\frac{J\left(u_{k}\right)}{\left\|u_{k}\right\|_{2}^{2}}\right)^{\alpha}\left\|u_{k}\right\|_{2}^{2}-\left(\frac{J\left(u_{k}\right)}{\left\|p_{k}\right\|_{2}^{2}}\right)^{1-\alpha} J\left(u_{k}\right) \\
& =\left(J\left(u_{k}\right)\right)^{\alpha}\left(\left\|u_{k}\right\|_{2}^{2-2 \alpha}-\frac{\left(J\left(u_{k}\right)\right)^{2-2 \alpha}}{\|p\|_{2}^{2-2 \alpha}}\right) \\
& \geq 0
\end{aligned}
$$

since $J\left(u_{k}\right) \leq\left\|u_{k}\right\|_{2} \cdot\left\|p_{k}\right\|_{2}$. From (19) and (20), we get

$$
\left\|u_{k+1}\right\|_{2} \geq\left\|u_{k}\right\|_{2} .
$$

Notice that we can also deduce from relations (15), (19) and (20) that

$$
\begin{aligned}
\left\|u_{k+1}\right\|_{2}^{2} & \geq\left\|u_{k}\right\|_{2}^{2}+(\delta t)^{2}\left\|\beta_{k} u_{k+1}-\gamma_{k} p_{k+1}\right\|_{2}^{2} \\
\left\|u_{k+1}\right\|_{2}^{2} & \geq\left\|u_{k}\right\|_{2}^{2}+\left\|u_{k+1}-u_{k}\right\|_{2}^{2} \\
2\left\langle u_{k+1}, u_{k}\right\rangle & \geq 2\left\|u_{k}\right\|_{2}^{2}
\end{aligned}
$$

so that $\left\langle u_{k+1}, u_{k}\right\rangle \leq\left\|u_{k+1}\right\|_{2}\left\|u_{k}\right\|_{2} \leq\left\|u_{k+1}\right\|_{2}^{2}$. 
4 The optimality conditions of the minimizer of (16) state that there exists $p_{k+1} \in \partial J\left(u^{k+1}\right)$ such that $\frac{1}{\gamma_{k} \delta t}\left(u_{k+1}-u_{k}\right)-\frac{\beta_{k}}{\gamma_{k}} u_{k+1}+p_{k+1}=0$, which gives

$$
p_{k+1}=\frac{1}{\gamma_{k} \delta t} u_{k}-\frac{1}{\gamma_{k}}\left(\frac{1}{\delta t}-\beta_{k}\right) u_{k+1}:=\mu u_{k}-\nu u_{k+1},
$$

with $\mu \geq \nu \geq 0$. Taking the scalar product of (23) with $p^{k+1}$, we have:

$$
\begin{aligned}
\left\langle p^{k+1}, p^{k+1}\right\rangle & =\mu\left\langle u^{k}, p^{k+1}\right\rangle-\nu\left\langle u^{k+1}, p^{k+1}\right\rangle \\
\left\|p^{k+1}\right\|_{2}^{2} & =\mu\left\langle u^{k}, p^{k+1}\right\rangle-\nu J\left(u^{k+1}\right) \\
\left\|p^{k+1}\right\|_{2}^{2}+\nu J\left(u^{k+1}\right) & \leq \mu J\left(u^{k}\right),
\end{aligned}
$$

where we observe that $\left\langle u^{k}, p^{k+1}\right\rangle \geq 0$. Next, by taking the scalar product of $(23)$ with any $p^{k} \in \partial J\left(u_{k}\right)$, we have

$$
\begin{aligned}
\left\langle p^{k+1}, p^{k}\right\rangle & =\mu\left\langle u^{k}, p^{k}\right\rangle-\nu\left\langle u^{k+1}, p^{k}\right\rangle \\
\mu J\left(u^{k}\right) & \leq\left\langle p^{k+1}, p^{k}\right\rangle+\nu\left\langle J\left(u^{k+1}\right) .\right.
\end{aligned}
$$

By (24) and (25) we get $\left\|p^{k+1}\right\|_{2}^{2} \leq\left\langle p^{k+1}, p^{k}\right\rangle$ so that $\left\|p^{k+1}\right\|_{2} \leq\left\|p^{k}\right\|_{2}$.

\section{Corollary 1. If}

$$
\frac{1}{\delta t}>\left(\frac{J\left(u_{0}\right)}{\left\|u_{0}\right\|_{2}^{2}}\right)^{\alpha}
$$

then the assumption $\frac{1}{\delta t}>\left(\frac{J\left(u_{k}\right)}{\left\|u_{k}\right\|_{2}^{2}}\right)^{\alpha}$ of Proposition 5 is valid $\forall k \geq 0$.

Proof. Let us assume that $\frac{1}{\delta t}>\left(\frac{J\left(u_{0}\right)}{\left\|u_{0}\right\|_{2}^{2}}\right)^{\alpha}$. Make the induction hypothesis that $\frac{1}{\delta t}>\left(\frac{J\left(u_{k}\right)}{\left\|u_{k}\right\|_{2}^{2}}\right)^{\alpha}$, for all $k \leq N$. Then to prove that it still holds for $N+1$, one just need to notice that from relations (17) and (21), we have

$$
\begin{aligned}
\frac{J\left(u_{N+1}\right)}{\left\|u_{N+1}\right\|_{2}} & \leq \frac{J\left(u_{0}\right)}{\left\|u_{0}\right\|_{2}} \\
\frac{J\left(u_{N+1}\right)}{\left\|u_{N+1}\right\|_{2}^{2}} & \leq \frac{J\left(u_{0}\right)}{\left\|u_{0}\right\|_{2}^{2}},
\end{aligned}
$$

so that

$$
\left(\frac{J\left(u_{N+1}\right)}{\left\|u_{N+1}\right\|_{2}^{2}}\right)^{\alpha} \leq\left(\frac{J\left(u_{0}\right)}{\left\|u_{0}\right\|_{2}^{2}}\right)^{\alpha}<\frac{1}{\delta t}
$$




\section{The case $\alpha=1$}

From now, we will assume that $\left\|u_{0}\right\|_{2}=1$ and we will restrict our attention to the case when $\alpha=1$, where the flow (13) is:

$$
\left\{\begin{array}{l}
u(0)=u_{0}, \\
u_{t}=\frac{J(u)}{\|u\|_{2}^{2}} u-p, \quad p \in \partial J(u) .
\end{array}\right.
$$

This flow may be easier to analyze since we get rid of $\|p\|_{2}$, while keeping constant $\|u(t)\|_{2}$. Observing that $J(u)=\langle p, u\rangle$ so that $u_{t}=\left\langle p, \frac{u}{\|u\|_{2}}\right\rangle \frac{u}{\|u\|_{2}}-p$, the behaviour of this flow is illustrated in Figure 2. The PDE makes $u$ evolve on the boundary of an $\ell_{2}$ ball of radius $\left\|x_{0}\right\|_{2}$ (we assume that $\left\|x_{0}\right\|_{2}>C$ defined in (4)) until there exists a subgradient $p \in \partial J(u) \subset K$ such that $p$ is the orthogonal projection of $u$ onto $K$. As characterized in Proposition 1, an eigenfunction is thus obtained as soon as $p \in \partial J(u)$ and $p=\operatorname{Proj}_{K}(u)$.
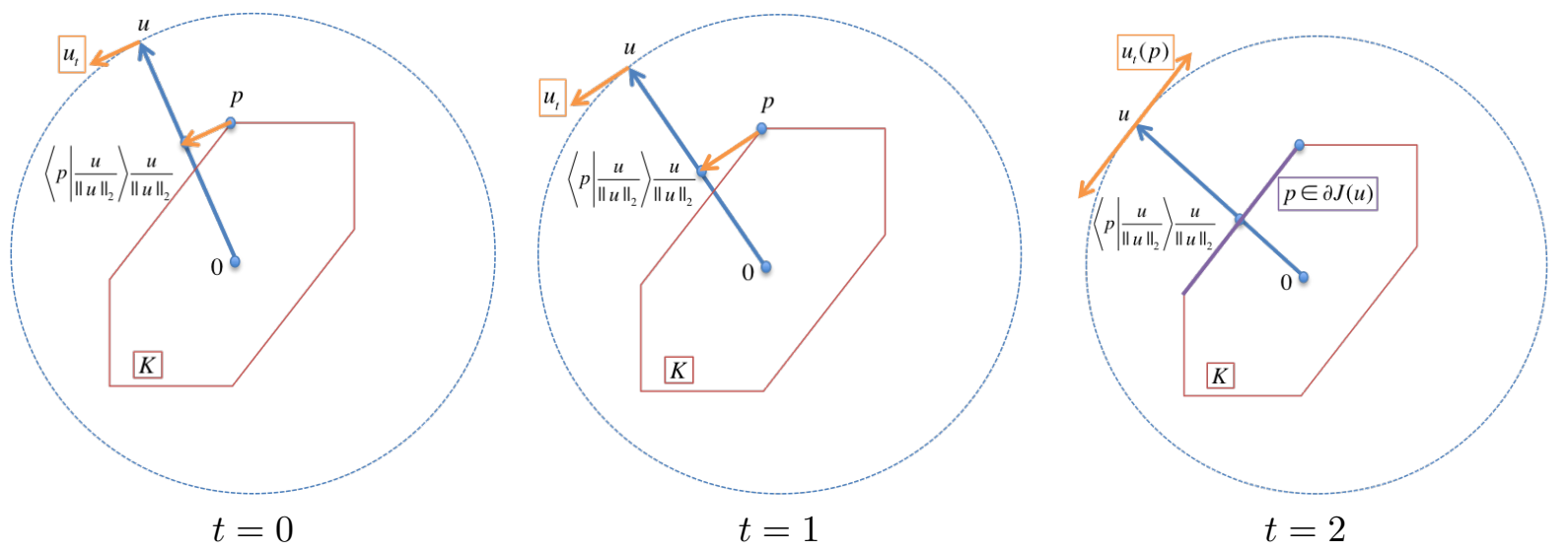

Figure 2: Illustration of the evolution of $u(t)$ with an explicit discretization of the flow (27). The vector $p \in \partial J(u)$ and its projection onto $u$ gives the direction $u_{t}$ of the flow.

\subsection{Uniqueness of a solution of (27)}

We start our analysis of the flow by stating a comparison result.

Proposition 6. Let $u$ and $v$ be two solutions of (27) with respective initial condition $u_{0}$ and $v_{0}$ such that $J\left(u_{0}\right)<+\infty$ and $J\left(v_{0}\right)<+\infty$, with $\left\|u_{0}\right\|_{2}=$ 
$\left\|v_{0}\right\|_{2}=1$. Then we have:

$$
\frac{d}{d t}\left(\frac{1}{2}\|u-v\|_{2}^{2}\right) \leq \frac{J(u)+J(v)}{2}\|u-v\|_{2}^{2}
$$

Uniqueness of a solution for the flow is then a direct consequence, as stated in the next corollary.

Corollary 2. Let $u$ and $v$ be two solutions of (27) with respective initial condition $u_{0}$ and $v_{0}$, such that $J\left(u_{0}\right)<+\infty$ and $J\left(v_{0}\right)<+\infty$, with $\left\|u_{0}\right\|_{2}=$ $\left\|v_{0}\right\|_{2}=1$. Then we have:

$$
\frac{d}{d t}\left(\frac{1}{2}\|u-v\|_{2}^{2}\right) \leq \frac{J\left(u_{0}\right)+J\left(v_{0}\right)}{2}\|u-v\|_{2}^{2}
$$

Moreover, we have:

$$
\|u-v\|_{2}^{2} \leq\left\|u_{0}-v_{0}\right\|_{2}^{2} \exp \left(\left(J\left(u_{0}\right)+J\left(v_{0}\right)\left(t-t_{0}\right)\right)\right.
$$

The corollary is a direct consequence of the previous proposition, the fact that $J(u)$ is decreasing, and Gronwall lemma. Let us now prove the proposition.

Proof. From the properties of the flow, we have $\|u\|_{2}=\|v\|_{2}=1$. Moreover, we have $J(u) \leq J\left(u_{0}\right)$ and $J(v) \leq J\left(v_{0}\right)$ for all $t$. Let us compute:

$$
\begin{aligned}
\frac{d}{d t}\left(\frac{1}{2}\|u-v\|_{2}^{2}\right) & =\left\langle u-v, u_{t}-v_{t}\right\rangle \\
& =\langle J(u) u-p-J(v) v+q, u-v\rangle, \quad p \in \partial J(u) q \in \partial J(v) \\
& =\langle J(u) u-J(v) v, u-v\rangle+\langle q-p, u-v\rangle, \quad p \in \partial J(u) q \in \partial J(v)
\end{aligned}
$$

But $\langle q-p, u-v\rangle \leq 0$ since $p \in \partial J(u)$ and $q \in \partial J(v)$ (as $J$ is convex). Hence:

$$
\frac{d}{d t}\left(\frac{1}{2}\|u-v\|^{2}\right) \leq\langle J(u) u-J(v) v, u-v\rangle
$$

But:

$$
\begin{aligned}
\langle J(u) u-J(v) v, u-v\rangle & =-J(u)\langle u, v\rangle+J(u)+J(v)-J(v)\langle u, v\rangle \\
& =(J(u)+J(v))(1-\langle u, v\rangle) \\
& =\frac{J(u)+J(v)}{2}\|u-v\|_{2}^{2}
\end{aligned}
$$

So that we get (28).

Notice of course that uniqueness of the solution of (27) comes at once from (30). 


\subsection{Properties of a semi-explicit scheme}

For $\alpha=1$, the numerical scheme (14) becomes:

$$
\frac{u_{k+1}-u_{k}}{\delta t}=\frac{J\left(u_{k}\right) u_{k+1}}{\left\|u_{k}\right\|_{2}^{2}}-p_{k+1}
$$

associated with the minimization of

$$
F\left(u, u_{k}\right)=\frac{1}{2 \delta t}\left\|u-u_{k}\right\|_{2}^{2}-\frac{J\left(u_{k}\right)}{2\left\|u_{k}\right\|_{2}^{2}}\|u\|_{2}^{2}+J(u) .
$$

$u_{k+1}$ is a minimizer of $F\left(., u_{k}\right)$, as soon as $\frac{1}{\delta t}>\frac{J\left(u_{0}\right)}{\left\|u_{0}\right\|_{2}^{2}} \geq \frac{J\left(u_{k}\right)}{\left\|u_{k}\right\|_{2}^{2}}$. From Proposition 4 , the continuous flow keeps $\|u\|_{2}$ constant for $\alpha=1$, but the discrete properties studied in Proposition 5 just ensure that $\left\|u_{k}\right\|_{2}$ is non decreasing. As a consequence, instead of dealing with (33), we consider the following renormalization to ensure that for $\left\|u_{0}\right\|_{2}=1,\left\|u_{k}\right\|_{2}=1, \forall k>0$ :

$$
\begin{cases}\frac{u_{k+1 / 2}-u_{k}}{\delta t} & =J\left(u_{k}\right) u_{k+1 / 2}-p_{k+1 / 2}, p_{k+1 / 2} \in \partial J\left(u_{k+1 / 2}\right) \\ u_{k+1} & =\frac{u_{k+1 / 2}}{\left\|u_{k+1 / 2}\right\|_{2}} .\end{cases}
$$

This scheme is associated with the minimization of

$$
\tilde{F}\left(u, u_{k}\right)=\frac{1}{2 \delta t}\left\|u-u_{k}\right\|_{2}^{2}-\frac{J\left(u_{k}\right)}{2}\|u\|_{2}^{2}+J(u)+\chi_{\|\cdot\|_{2} \leq 1}(u) .
$$

Proposition 7. $u_{k+1}$ defined in (34) is the minimizer of $\tilde{F}$.

Proof. We define $u_{k+1}$ as the minimizer of (35).

Then:

$$
0 \in \frac{u_{k+1}-u_{k}}{\delta t}-J\left(u_{k}\right) u_{k+1}+p_{k+1}+\partial \chi_{\|\cdot\|_{2} \leq 1}\left(u_{k+1}\right)
$$

So that:

$$
u_{k}-\delta t p_{k+1} \in u_{k+1}\left(1-\delta t J\left(u_{k}\right)\right)+\delta t \partial \chi_{\|\cdot\|_{2} \leq 1}\left(u_{k+1}\right)
$$

Hence

$$
u_{k+1}=\left(I d+\frac{\delta t}{1-\delta t J\left(u_{k}\right)} \partial \chi_{\|\cdot\|_{2} \leq 1}\right)^{-1}\left(\frac{1}{1-\delta t J\left(u_{k}\right)}\left(u_{k}-\delta t p_{k+1}\right)\right)
$$

We deduce that $u_{k+1}$ is the $L^{2}$ projection on the ball of radius 1 of

$$
\frac{1}{1-\delta t J\left(u_{k}\right)}\left(u_{k}-\delta t p_{k+1}\right)
$$


But from (32), since $\left\|u_{k}\right\|_{2}=1$, we know that:

$$
u_{k+1 / 2}=\frac{u_{k}-\delta t p_{k+1 / 2}}{1-\delta t J\left(u_{k}\right)}
$$

So we see that $u_{k+1}$ is the $L^{2}$ projection on the ball of radius 1 of $u_{k+1 / 2}$. Moreover, since the scheme defined by (32) is such that $\left\|u_{k+1 / 2}\right\|_{2} \geq\left\|u_{k}\right\|_{2}$, we deduce that $\left\|u_{k+1 / 2}\right\|_{2} \geq 1$, and thus

$$
u_{k+1}=\frac{u_{k+1 / 2}}{\left\|u_{k+1 / 2}\right\|_{2}}
$$

Hence $u_{k+1}$ is also solution of (34).

Thanks to Proposition (7), we are now in position to analyse the sequence $u_{k}$ defined by $(34)$.

Theorem 1. Let $u_{0}$ in $X$, and the sequence $u_{k}$ defined by (34). Then the sequences $J\left(u_{k}\right)$ and $\left\|p_{k}\right\|_{2}$ are non increasing, $\left\|u_{k}\right\|_{2}=\left\|u_{0}\right\|_{2}$ for all $k$, and $u_{k+1}-u_{k} \rightarrow 0$.

Proof. $\left\|u_{k}\right\|_{2}$ constant. Let us proceed by contradiction and assume that $\left\|u_{k}\right\|_{2}=1$ and $\left\|u_{k+1}\right\|_{2}<1$, where $u_{k+1}$ is the minimizer of (35). As the constraint $\chi_{\|\cdot\|_{2} \leq 1}\left(u_{k+1}\right)$ is not saturated then

$$
u_{k+1}=\underset{u}{\operatorname{argmin}} \frac{1}{2 \delta t}\left\|u-u_{k}\right\|_{2}^{2}-\frac{J\left(u_{k}\right)}{2}\|u\|_{2}^{2}+J(u),
$$

which means that $1>\left\|u_{k+1}\right\|_{2} \geq\left\|u_{k}\right\|_{2}=1$ from Proposition 5, which is impossible.

$J\left(u_{k}\right)$ and $\left\|p_{k}\right\|_{2}$ non increasing. Since $\left\|u_{k}\right\|_{2}=\left\|u_{0}\right\|_{2}=1$, we can use Proposition 5 with $\alpha=1$ and therefore conclude.

Convergence of $u_{k+1}-u_{k}$. Assume that $J\left(u_{0}\right)<\frac{1}{\delta t}$ to have $\tilde{F}$ convex, and $\left\|u_{k}\right\|_{2}=1$, then:

$$
\begin{aligned}
\tilde{F}\left(u_{k+1}, u_{k}\right) & \leq \tilde{F}\left(u_{k}, u_{k}\right) \\
\frac{1}{2 \delta t}\left\|u_{k+1}-u_{k}\right\|_{2}^{2}-\frac{J\left(u_{k}\right)}{2}\left\|u_{k+1}\right\|_{2}^{2}+J\left(u_{k+1}\right) & \leq \frac{J\left(u_{k}\right)}{2} \\
\frac{1}{2 \delta t}\left\|u_{k+1}-u_{k}\right\|_{2}^{2}+J\left(u_{k+1}\right) & \leq \frac{J\left(u_{k}\right)}{2}\left(1+\left\|u_{k+1}\right\|_{2}^{2}\right) \\
\frac{1}{2 \delta t}\left\|u_{k+1}-u_{k}\right\|_{2}^{2}+J\left(u_{k+1}\right) & \leq J\left(u_{k}\right),
\end{aligned}
$$


since $\left\|u_{k+1}\right\|_{2} \leq 1$.

Summing on $k$ from 0 to $N-1$ relation (42), we deduce that:

$$
\frac{1}{2 \delta t} \sum_{k=1}^{N-1}\left\|u_{k+1}-u_{k}\right\|_{2}^{2} \leq J\left(u_{0}\right)-J\left(u_{N}\right) \leq J\left(u_{0}\right)
$$

In particular $\sum_{k}\left\|u_{k+1}-u_{k}\right\|_{2}^{2}$ converges, and $u_{k+1}-u_{k} \rightarrow 0$.

\subsection{Convergence of the semi-implicit scheme}

We are now in position to state some convergence results. We first consider the case when $X$ is a finite dimensional space. We will then consider the general case when $X$ is an infinite dimensional case, for which we need to add a technical hypothesis to get a convergence result.

\subsubsection{Finite dimensional case}

Theorem 2. Let $u_{0}$ in $X$, with $X$ of finite dimension, and the sequence $u_{k}$ defined by (34). There exists some $u$ and $p$ in $X$ such that up to a subsequence, $u_{k}$ converges to $u$ in $X$ and $p_{k}$ converges to $p$ in $X$, with $p \in$ $\partial J(u)$, and $J\left(u_{k}\right)$ converges to $J(u)$. Moreover, $u$ satisfies the differential inclusion:

$$
J(u) u-u-p \in \partial \chi_{\|\cdot\|_{2} \leq 1}(u)
$$

Proof. From Theorem 1, there exists $u$ in $X$ such that up to a subsequence, $u_{k} \rightarrow u$ in $X$. There exists also $p$ in $X$ such that up to a subsequence, $p_{k} \rightarrow p$ in $X$.

Let $v$ in $X$. We have:

$$
J(v) \geq J\left(u_{k}\right)+\left\langle v-u_{k}, p_{k}\right\rangle
$$

We can let $k \rightarrow+\infty$ and using the lower semi continuity of $J$ we get:

$$
J(v) \geq J(u)+\langle v-u, p\rangle
$$

Hence $p \in \partial J(u)$.

Moreover, we have $J\left(u_{k}\right)=\left\langle u_{k}, p_{k}\right\rangle$. Letting again $k \rightarrow+\infty$, we se that $J\left(u_{k}\right) \rightarrow J(u)=\langle u, p\rangle$.

Let again $v$ in $X$. We have, for $p_{k+1}$ in $\partial J\left(u_{k+1}\right)$ :

$$
-\frac{u_{k+1}-u_{k}}{\delta t}+J\left(u_{k+1}\right) u_{k+1}-p_{k+1}+\in \partial \chi_{\|\cdot\|_{2} \leq 1}\left(u_{k+1}\right) .
$$


Hence:

$$
\chi_{\|\cdot\|_{2} \leq 1}(v) \geq \chi_{\|\cdot\|_{2} \leq 1}\left(u_{k+1}\right)+\left\langle v-u_{k+1},-\frac{u_{k+1}-u_{k}}{\delta t}+J\left(u_{k}\right) u_{k+1}-p_{k+1}\right\rangle
$$

We can let $k \rightarrow+\infty$ so that:

$$
\chi_{\|\cdot\|_{2} \leq 1}(v) \geq \chi_{\|\cdot\|_{2} \leq 1}(u)+\langle v-u, J(u) u-p\rangle .
$$

We thus deduce that (44) holds.

\subsubsection{Infinite dimensional case}

In this case, we consider that $J$ is defined on $X:=L^{2}(\Omega)$. We first state a preliminary convergence result.

Proposition 8. Let $u_{0}$ in $L^{2}(\Omega)$, and the sequence $u_{k}$ defined by (34). There exists some $u$ and $p$ in $X$ such that up to a subsequence, $u_{k}$ converges to $u$ and $p_{k}$ converges to $p$ in $L^{2}(\Omega)$ weak.

Proof. Since $\left\|u_{k}\right\|_{2}=1$ for all $k, u_{k}$ is a bounded sequence in $L^{2}(\Omega)$. Hence (see e.g. [17]) there exists $u$ in $L^{2}(\Omega)$ such that up to a subsequence, $u_{k} \rightarrow u$ in $L^{2}(\Omega)$ weak. With the same reasoning, we can show that there exists $p$ in $L^{2}(\Omega)$ such that up to a subsequence, $p_{k} \rightarrow p$ in $L^{2}(\Omega)$ weak.

To state a full convergence result, we need to add a technical hypothesis.

Theorem 3. Let $u_{0}$ in $L^{2}(\Omega)$, and the sequence $u_{k}$ defined by (34). Assume that the sequence $u_{k}$ lives in a compact subset of $L^{2}(\Omega)$ for the strong topology. There exists some $u$ and $p$ in $L^{2}(\Omega)$ such that up to a subsequence, $u_{k}$ converges to $u$ in $L^{2}(\Omega)$ strong and $p_{k}$ converges to $p$ in $L^{2}(\Omega)$ weak, with $p \in \partial J(u)$, and $J\left(u_{k}\right)$ converges to $J(u)$. Moreover, $u$ satisfies the differential inclusion:

$$
J(u) u-u-p \in \partial \chi_{\|\cdot\|_{2} \leq 1}(u)
$$

Proof. From the previous proposition, there exists $u$ in $L^{2}(\Omega)$ such that up to a subsequence, $u_{k} \rightarrow u$ in $L^{2}(\Omega)$ weak. There exists also $p$ in $L^{2}(\Omega)$ such that up to a subsequence, $p_{k} \rightarrow p$ in $L^{2}(\Omega)$ weak. Since $u_{k}$ lives in a compact subset of $L^{2}(\Omega)$ for the strong topology, we have that $u_{k} \rightarrow u$ in $L^{2}(\Omega)$ strong.

The rest of the proof is identical to the one of Theorem 2 , since $X:=$ $L^{2}(\Omega)$. 


\subsubsection{Eigenfunction}

The following result shows that the limit $u$ of the semi-implicit scheme is indeed an eigenfunction.

Proposition 9. If u satisfies either Equation 44 or Equation 50, then $u$ is an eigenfunction.

Proof. From either Equation 44 or Equation 50 it follows that there exists $p \in \partial J(u)$ and $q \in \partial \chi_{\|\cdot\|_{2} \leq 1}(u)$ such that: $-J(u) u+p+q=0$. Since $\|u\|_{2}=1$, we have $\partial \chi_{\|\cdot\|_{2} \leq 1}(u)=\{\gamma u, \gamma \geq 0\}$, and therefore:

$$
\begin{aligned}
-J(u) u+p+\gamma u & =0 \\
p & =(J(u)-\gamma) u
\end{aligned}
$$

and $u$ is an eigenfunction. Moreover, as $J(u)=\langle p, u\rangle$, we get $\gamma=0$.

Now that we have analyzed a semi-explicit scheme for computing an eigenfunction, we turn our attention to the time continuous problem (evolution equation) in the next section.

\subsection{Existence of solution for (27)}

In this section the time continuous flow is analyzed. Let us rewrite here (27):

$$
\left\{\begin{array}{l}
u(0)=u_{0}, \\
u_{t}=\frac{J(u)}{\|u\|_{2}^{2}} u-p, \quad p \in \partial J(u) .
\end{array}\right.
$$

We have the following existence and uniqueness result. As in the case of Theorem 3, we need to add a technical hypothesis.

Theorem 4. Let $u_{0}$ in $L^{2}(\Omega)$ with $J\left(u_{0}\right)<+\infty$. Assume that $\check{u}_{\delta t}$ lives in some compact set $K_{T}$ for the strong topology of $L^{2}\left((0, T) ; L^{2}(\Omega)\right)$. Then problem(51) admits exactly one solution in $W^{1,2}\left((0, T) ; L^{2}(\Omega)\right)$.

The proof of this theorem is detailed in Appendix A

\section{$5 \quad$ Numerical Results}

Here we give a few examples of running the flow in several settings. We first examine local TV regularizers. Fig. 3 shows the results using isotropic TV, 
where the gradient magnitude is based on $\ell^{2},|\nabla u|=\sqrt{\left(u_{x}\right)^{2}+\left(u_{y}\right)^{2}}$. On the top right and middle the results of $u$ and $p$, respectively, are shown after 100 iterations. The initialization can be noise or some image to produce different eigenfunctions, here we chose the cameraman image. It can be seen that $p$ is very similar to $u$ in its shape, which is expected for eigenfunctions. In the ideal case, we should expect $p=\lambda u$ pointwise, therefore for every pixel the ratio $p / u=\lambda$ should yield spatially a constant image. In Fig. 3 top right this ratio is shown, where most of the image is of constant value, but there are some deviations near the boundaries of the shape. There is still no definitive theory of eigenfunctions of discrete isotropic TV. Our experiments indicate that numerically one reaches in general only approximations of eigenfunctions of the continuous case. Convergence to precise eigenfunctions are reached in trivial cases, such as partitions by straight lines of the space. Consequently, the process is very stable when $p$ and $u$ are very similar, but full convergence is not attained numerically, as can be seen in Fig. 3 bottom.

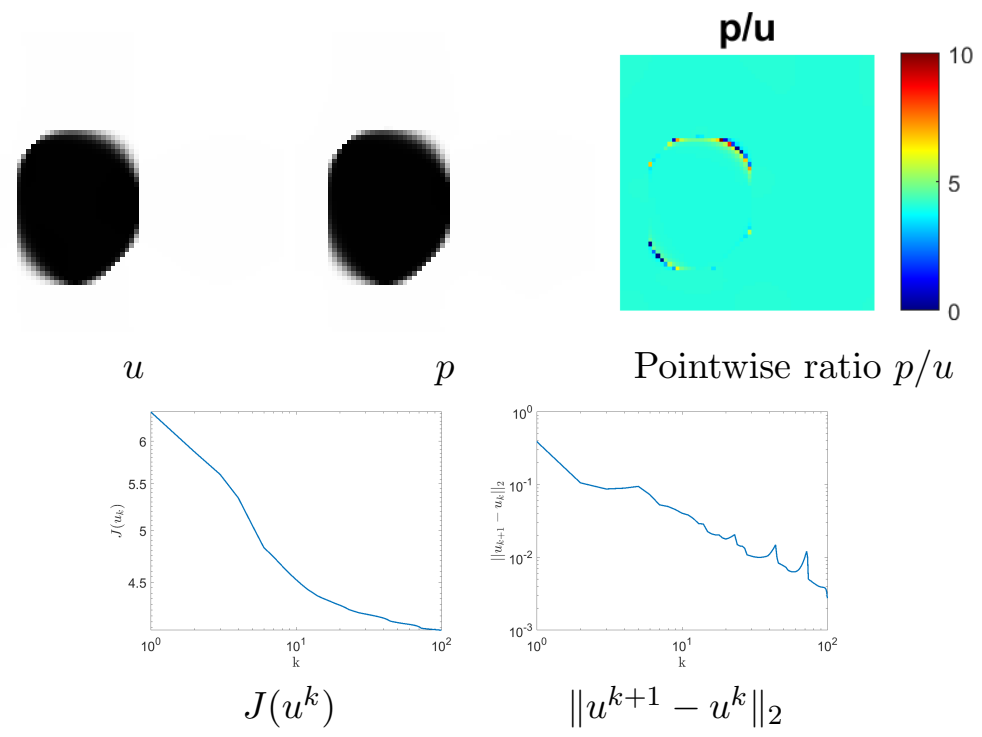

Figure 3: Results of the flow for isotropic TV. Top: $u$ and $p$ and the ratio $u / p$ after 100 iterations (the ratio should be close to a constant function). Bottom, the values of $J\left(u^{k}\right)$ and $\left\|u^{k+1}-u^{k}\right\|$ are plotted as a function of iterations $k$. 
Fig. 4 shows the case of local anisotropic TV, where the gradient magnitude is based on $\ell^{1},|\nabla u|=\left|u_{x}\right|+\left|u_{y}\right|$. In this case, $u$ and $p$ have exactly the same shape, and the ratio $p / u$ (top-right) is constant, up to numerical precision. As we reach a precise eigenfunction the algorithm fully converges to a steady state, as seen (bottom) on the values of $J\left(u^{k}\right)$ and $\left\|u^{k+1}-u^{k}\right\|_{2}$ as a function of the iteration $k$. These experiments are useful to examine the algorithm and to compute local discrete TV eigenfunctions.

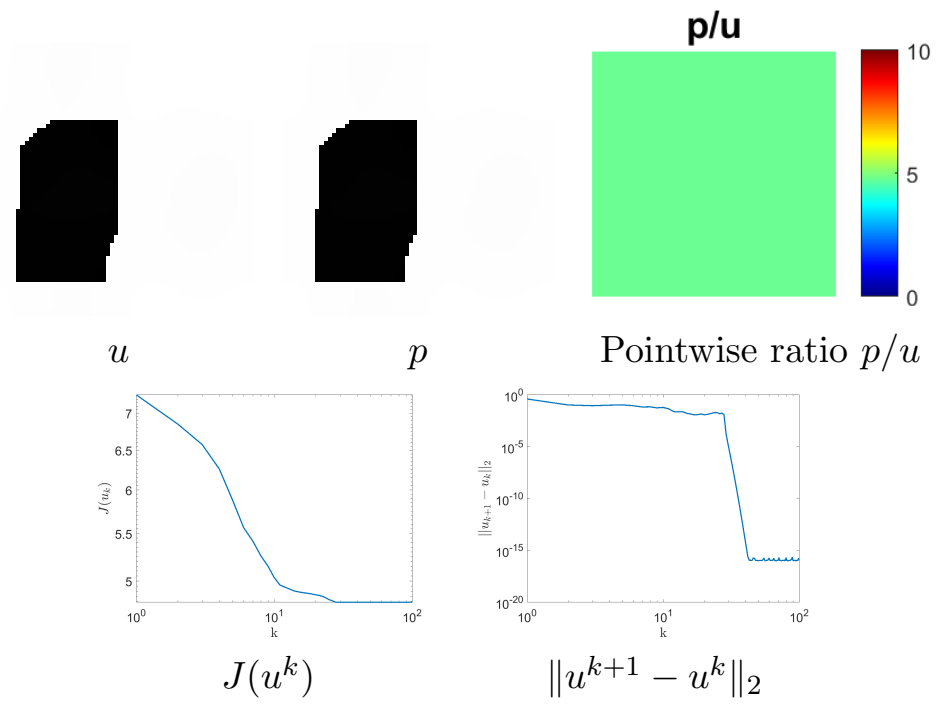

Figure 4: Results of the flow for anisotropic TV. Top: $u$ and $p$ and the ratio $u / p$ after 100 iterations. Bottom, the value of $J$ and $\left\|u^{k+1}-u^{k}\right\|$ as a function of iterations $k$. One can observe the process reached complete convergence, where $u$ is a precise eigenfunction.

For segmentation and clustering purposes, TV on graphs is used as the regularizer of choice. One constructs a graph based on the input data and computes the iterative flow. We use $J$ as defined in (3) with $q=1$ where $w_{i j}$ is the weight of the graph between node $i$ and node $j$. We give examples of graph based on an image, for segmentation, and one based on point cloud, for clustering. In Fig. 5 the graph is constructed from the image based on Euclidean patch-distances, as for instance in [27]. We use a $5 \times 5$ search window for similar patches, so the pixel proximity relation is essentially very local. Our initialization of the flow is the input image $f$ (left). We show the result of $u$ after 50 iterations. We see that the process naturally converges to a segmentation of the data (see thresholded result on the right). 

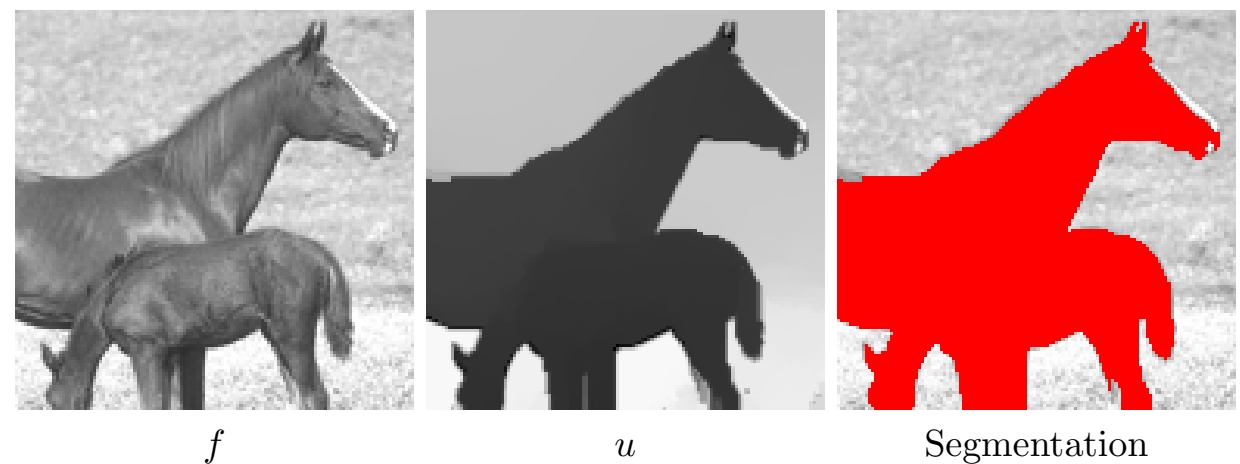

Figure 5: Results of the flow for TV defined on graphs constructed from the image, based on patch distances. Initializing with the input image, the flow yields a segmentation of the image.

In Fig. 6 a graph is constructed from the 2D point cloud, where weights are computed based on local Euclidean distance. The flow is initialized randomly and converges to the natural clustering of the data.

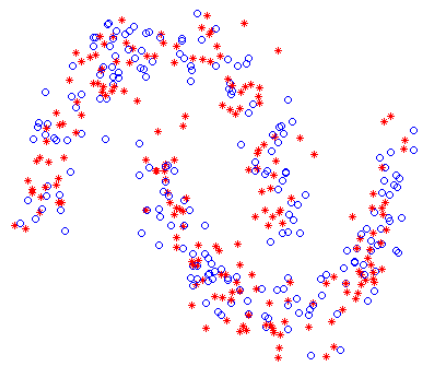

Initialization.

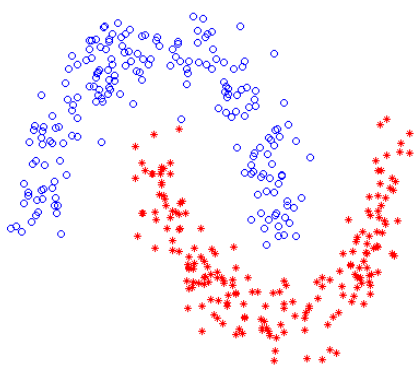

Converged state.

Figure 6: Results of the flow for TV defined on graphs based on point cloud distances. The processes converges to natural clustering of the data. 


\section{Conclusion}

In this work we have presented a class of nonlinear flows for which their steady-states are eigenfunctions with respect to the subgradient of a desired one-homogeneous regularization functional, such as any flavor of totalvariation on grids or graphs.

The flows were analyzed in finite dimensions both in the continuous time setting and in the discrete setting. The discrete setting is realized as a series of convex optimization iterations. Its properties and stability characteristics were shown. For a specific case of the proposed $\alpha$-flow, a comprehensive theory was derived. It was shown that the discrete iterations converge to a steady state, which is an eigenfunction. Moreover, we have shown that the time continuous flow exists and has a unique solution.

These algorithms can be used for several applications related to segmentation and clustering, where graph total variation and eigenfunctions of the 1-Laplacian operator are used (see e.g. $[14,15,18]$ ). The flows are continuously evolving towards an eigenfunction and are very convenient to use when one has a rough initial estimate (for instance, using linear eigenfunctions as approximations). Future research will include further examination of the general continuous case. In addition, we will examine the simultaneous evolution of several flows to compute several eigenfunctions, e.g. for multi-class clustering applications.

Acknowledgements The authors would like to thank Antonin Chambolle for many discussions related to this work. This study has been carried out with financial support from the French State, managed by the French

National Research Agency (ANR GOTMI) (ANR-16-CE33-0010-01). JeanFranois Aujol is a member of Institut Universitaire de France (IUF). Guy Gilboa acknowledges support by the Israel Science Foundation (grant No. 718/15). This study as been carried out with financial support from EU RISE project NoMADS. 


\section{A Existence of a solution for (27)}

To show that problem (27) has a solution, we start from the semi-discrete problem (34). Thanks to Proposition (7), we therefore consider a sequence $\left(u_{k}\right)$ satisfying $(35)$. We know that $\left(u_{k}\right)$ satisfies:

$$
-\frac{u_{k+1}-u_{k}}{\delta t}+J\left(u_{k+1}\right) u_{k+1}-p_{k+1} \in \partial \chi_{\left\|_{\cdot}\right\|_{2} \leq 1}\left(u_{k+1}\right)
$$

with $p_{k+1}$ in $\partial J\left(u_{k+1}\right)$. From the result of Section (4), we know that the sequence $\left(u_{k}\right)$ exists and is unique provided $\delta t$ small enough.

\section{A.1 Definitions of interpolate functions}

For $t_{0}=0$ and $t_{k}=k \delta t$, we classically introduce two piecewise constant functions defined on $\Omega \times \mathbb{R}^{+}$(see e.g. [6, 4]):

$$
\begin{aligned}
& \check{u}_{\delta t}(t, x)=u_{[t / \delta t]+1}(x)=u_{k+1}(x) \text { if } t_{k}<t \leq t_{k+1} \\
& \check{p}_{\delta t}(t, x)=p_{[t / \delta t]+1}(x)=p_{k+1}(x) \text { if } t_{k}<t \leq t_{k+1},
\end{aligned}
$$

where $[t / \delta t]$ is the integer part of $t / \delta t$.

We also introduce:

$$
\hat{u}_{\delta t}(t, x)=\left(t-t_{n}\right) \frac{u_{k+1}(x)-u_{k}(x)}{\delta t}+u_{k}(x)
$$

with $k=[t / \delta t] . \hat{u}_{\delta t}(., x)$ is piecewise affine, continuous, and we have:

$$
\frac{\partial \hat{u}_{\delta t}}{\partial t}(t, x)=\frac{u_{k+1}(x)-u_{k}(x)}{\delta t}, t_{k}<t<t_{k+1}
$$

With these notations, we can rewrite (52) as:

$\left.\left.-\frac{\left.\check{u}_{\delta t}(t, x)\right)-\check{u}_{\delta t}(t-\delta t, x)}{\delta t}+J\left(\check{u}_{\delta t}(t, x)\right) \check{u}_{\delta t}(t, x)\right)-\check{p}_{\delta t}(t, x) \in \partial \chi_{\|\cdot\|_{2} \leq 1}\left(\check{u}_{\delta t}(t, x)\right)\right)$

i.e.:

$$
\left.\left.-\frac{\partial \hat{u}_{\delta t}}{\partial t}(t, x)+J\left(\check{u}_{\delta t}(t, x)\right) \check{u}_{\delta t}(t, x)\right)-\check{p}_{\delta t}(t, x) \in \partial \chi_{\|\cdot\|_{2} \leq 1}\left(\check{u}_{\delta t}(t, x)\right)\right)
$$




\section{A.2 A priori estimates}

We first need to show some a priori estimates.

Proposition 10. Let $u_{0}$ in $L^{2}(\Omega)$. Then $t \mapsto J\left(\check{u}_{\delta t}(t,).\right)$, and $t \mapsto\left\|\check{p}_{\delta t}(t, .)\right\|_{2}$ are non increasing, $\left\|\check{u}_{\delta t}(t, .)\right\|_{2}=\left\|u_{0}\right\|_{2}$ for all $t$, and $\hat{u}_{\delta t}(t,).\left\|_{2} \leq 3\right\| u_{0} \|_{2}$.

Proof. This is a direct consequence of the previous section and equation (55).

Proposition 11. Let $T>0$ be fixed. There exists a constant $C>0$, which does not depend on $\delta t$, such that:

$$
\int_{0}^{T}\left\|\frac{\partial \hat{u}_{\delta t}}{\partial t}\right\|_{L^{2}(\Omega)}^{2} \leq C
$$

Proof. We have:

$$
\int_{t_{k}}^{t_{k+1}}\left\|\frac{\partial \hat{u}_{\delta t}}{\partial t}\right\|_{L^{2}(\Omega)}^{2}=\delta t \int_{\Omega}\left|\frac{u_{k+1}(x)-u_{k}(x)}{\delta t}\right|^{2} d x .
$$

By using (42), we get:

$$
\int_{t_{k}}^{t_{k+1}}\left\|\frac{\partial \hat{u}_{\delta t}}{\partial t}\right\|_{L^{2}(\Omega)}^{2} \leq 2\left(J\left(u_{k}\right)-J\left(u_{k+1}\right)\right) .
$$

Let us denote by $K=[T / \delta t]$, then

$$
\begin{aligned}
\sum_{n=0}^{K-1} \int_{t_{k}}^{t_{k+1}}\left\|\frac{\partial \hat{u}_{\delta t}}{\partial t}\right\|_{L^{2}(\Omega)}^{2} & \leq 2\left(J\left(u_{0}\right)-J\left(u_{K}\right)\right) \\
& \leq 2 J\left(u_{0}\right) .
\end{aligned}
$$

We thus deduce that:

$$
\int_{0}^{T}\left\|\frac{\partial \hat{u}_{\delta t}}{\partial t}\right\|_{L^{2}(\Omega)}^{2} d t \leq 2 T J\left(u_{0}\right)+\int_{t_{K}}^{T}\left\|\frac{\partial \hat{u}_{\delta t}}{\partial t}\right\|_{L^{2}(\Omega)}^{2} d t .
$$

But, by using (42), we have:

$$
\begin{aligned}
\int_{t_{K}}^{T}\left\|\frac{\partial \hat{u}_{\delta t}}{\partial t}\right\|_{L^{2}(\Omega)}^{2} d t & \leq 2 \frac{T-t_{K}}{\delta t}\left(J\left(u_{K}\right)-J\left(u_{K+1}\right)\right) \\
& \leq 2 J\left(u_{0}\right) .
\end{aligned}
$$


We then get from Proposition 10 that there exists $B>0$ which does not depend on $K$ and $\delta t$ such that: $\int_{t_{K}}^{T}\left\|\frac{\partial \hat{u}_{\delta t}}{\partial t}\right\|_{L^{2}(\Omega)}^{2} d t \leq B$. We then conclude thanks to (62).

Corollary 3. Let $T>0$ be fixed. Then:

$$
\lim _{\delta t \rightarrow 0} \int_{0}^{T}\left\|\hat{u}_{\delta t}-\check{u}_{\delta t}\right\|_{L^{2}(\Omega)}^{2} d t=0
$$

Proof. Let us denote by $K=[t / \delta t]$. We have:

$$
\int_{0}^{T}\left\|\hat{u}_{\delta t}-\check{u}_{\delta t}\right\|_{L^{2}(\Omega)}^{2} d t=\sum_{k=0}^{K-1} \int_{t_{k}}^{t_{k+1}}\left\|\hat{u}_{\delta t}-\check{u}_{\delta t}\right\|_{L^{2}(\Omega)}^{2} d t+\int_{t_{K}}^{T}\left\|\hat{u}_{\delta t}-\check{u}_{\delta t}\right\|_{L^{2}(\Omega)}^{2} d t,
$$

but:

$$
\sum_{k=0}^{K-1} \int_{t_{k}}^{t_{k+1}}\left\|\hat{u}_{\delta t}-\check{u}_{\delta t}\right\|_{L^{2}(\Omega)}^{2} d t=\sum_{k=0}^{K-1} \int_{t_{k}}^{t_{k+1}}\left\|\left(t-t_{k}-\delta t\right)\left(u_{k+1}-u_{k}\right)\right\|_{L^{2}(\Omega)}^{2} d t
$$

We then deduce from (56) that:

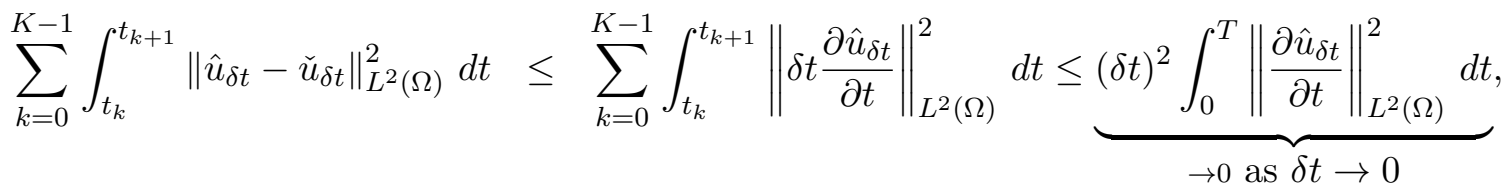

and:

$$
\int_{t_{K}}^{T}\left\|\hat{u}_{\delta t}-\check{u}_{\delta t}\right\|_{L^{2}(\Omega)}^{2} d t \leq \underbrace{(\delta t)^{3}\left\|\frac{u_{K+1}-u_{K}}{\delta t}\right\|_{L^{2}(\Omega)}^{2} d t}_{\rightarrow 0 \text { as } \delta t \rightarrow 0}
$$

\section{A.3 Convergence}

Theorem 4. Uniqueness of Theorem4 comes from Corollary 2 (see also e.g. $[16])$. Let us now prove existence of a solution. We first remark that, from Propositon 10 and 11, $\hat{u}_{\delta t}$ is uniformly bounded in $W^{1,2}\left((0, T) ; L^{2}(\Omega)\right)$, since $\|u\|_{W^{1,2}\left((0, T) ; L^{2}(\Omega)\right)}^{2}=\int_{0}^{T}\left\|\frac{\partial \hat{u}_{\delta t}}{\partial t}\right\|_{L^{2}(\Omega)}^{2}+\int_{0}^{T}\left\|\hat{u}_{\delta t}\right\|_{L^{2}(\Omega)}^{2}$. 
Thus, up to a subsequence, there exists $u$ in $W^{1,2}\left((0, T) ; L^{2}(\Omega)\right)$ such that $\hat{u}_{\delta t} \rightarrow u$ in $W^{1,2}\left((0, T) ; L^{2}(\Omega)\right)$ weak. Since $W^{1,2}\left((0, T) ; L^{2}(\Omega)\right)$ is compactly embedded in $L^{2}\left((0, T) ; L^{2}(\Omega)\right)$ (see [41], Theorem 2.1, chapter 3$), \hat{u}_{\delta t} \rightarrow u$ strongly in $L^{2}\left((0, T) ; L^{2}(\Omega)\right)$.

Since $\left\|\check{u}_{\delta t}\right\|_{2}=1$ for all $t \in(0, T)$, we have $\left\|\check{u}_{\delta t}\right\|_{L^{2}\left((0, T) ; L^{2}(\Omega)\right)}^{2}=\int_{0}^{T}\left\|\check{u}_{\delta t}\right\|_{L^{2}(\Omega)}^{2}=$ $T$ (thanks to Proposition 10). Hence $\check{u}_{\delta t}$ is a bounded sequence in $L^{2}\left((0, T) ; L^{2}(\Omega)\right)$. Thus there exists $\check{u}$ in $L^{2}\left((0, T) ; L^{2}(\Omega)\right)$ such that up to a subsequence, $\check{u}_{\delta t} \rightarrow \check{u}$ in $L^{2}\left((0, T) ; L^{2}(\Omega)\right)$. Since we assume that $\check{u}_{\delta t}$ lives in some compact set for the strong topology of $L^{2}\left((0, T) ; L^{2}(\Omega)\right)$, we deduce that $\check{u}_{\delta t} \rightarrow \check{u}$ in $L^{2}\left((0, T) ; L^{2}(\Omega)\right)$ strong. We can also show that there exists $p$ in $L^{2}\left((0, T) ; L^{2}(\Omega)\right)$ such that up to a subsequence, $\check{p}_{\delta t} \rightarrow p$ in $L^{2}\left((0, T) ; L^{2}(\Omega)\right)$ weak. From Proposition 3 , we deduce that $\breve{u}_{\delta t} \rightarrow u$ strongly in $L^{2}\left((0, T) ; L^{2}(\Omega)\right)$, so that $\check{u}=u$.

Let $v$ in $L^{2}(\Omega)$. We have:

$$
J(v) \geq J\left(\check{u}_{\delta t}\right)+\left\langle v-\check{u}_{\delta t}, \check{p}_{\delta t}\right\rangle .
$$

Let $\phi$ in $C_{c}^{0}(0, T)$ a test function, $\phi \geq 0$. We multiply (67) by $\phi$ and integrate on $(0, T)$ :

$$
\int_{0}^{T} J(v) \phi(t) d t \geq \int_{0}^{T} J\left(\check{u}_{\delta t}\right) \phi d t+\int_{0}^{T}\left\langle v-\check{u}_{\delta t}, \check{p}_{\delta t}\right\rangle \phi(t) d t
$$

i.e.

$$
\int_{0}^{T} J(v) \phi(t) d t \geq \int_{0}^{T} J\left(\check{u}_{\delta t}\right) \phi(t) d t+\int_{0}^{T} \int_{\Omega}\left(v-\check{u}_{\delta t}\right) \check{p}_{\delta t} \phi(t) d t d x .
$$

We want to let $\delta t \rightarrow 0$ in (69). By convexity, we have:

$$
\liminf \int_{0}^{T} J\left(\check{u}_{\delta t}\right) \phi(t) d t \geq \int_{0}^{T} J(u) \phi(t) d t .
$$

Now, since $\check{u}_{\delta t} \rightarrow u$ strongly in $L^{2}\left((0, T) ; L^{2}(\Omega)\right)$ and $\check{p}_{\delta t} \rightarrow p$ in $L^{2}\left((0, T) ; L^{2}(\Omega)\right)$ strong, the second term on the right hand-side of (69) tends to

$$
\int_{0}^{T} \int_{\Omega}(v-u) p \phi(t) d t d x .
$$

We thus get:

$$
\int_{0}^{T} J(v) \phi(t) d t \geq \int_{0}^{T} J(u) \phi(t) d t+\int_{0}^{T} \int_{\Omega}(v-u) p \phi(t) d t d x .
$$


This inequality holds for all $\phi \geq 0$, we deduce that for a.e. $t$ in $(0, T)$ :

$$
J(v) \geq J(u)+\int_{\Omega}(v-u) p d x .
$$

Hence $p \in \partial J(u)$.

Moreover, we have $J\left(\check{u}_{\delta t}\right)=\left\langle\check{u}_{\delta t}, \check{p}_{\delta t}\right\rangle$. Letting again $\delta t \rightarrow 0$, we see that $J\left(u_{\delta t}\right) \rightarrow J(u)=\langle u, p\rangle$. The semi-discrete implicit scheme writes for $\check{p}_{\delta t}$ in $\partial J\left(u_{\check{u}_{\delta t}}\right)$ and for a.e. $t \in(0, T)$ :

$$
\left.\left.-\frac{\partial \hat{u}_{\delta t}}{\partial t}(t, x)+J\left(\check{u}_{\delta t}(t, x)\right) \check{u}_{\delta t}(t, x)\right)-\check{p}_{\delta t}(t, x) \in \partial \chi_{\|\cdot\|_{2} \leq 1}\left(\check{u}_{\delta t}(t, x)\right)\right),
$$

We thus have for all $v$ in $L^{2}(\Omega)$, and a.e. $t \in(0, T)$ :

$$
\chi_{\|\cdot\|_{2} \leq 1}(v) \geq \chi_{\|\cdot\|_{2} \leq 1}\left(\check{u}_{\delta t}\right)+\left\langle v-\check{u}_{\delta t},-\frac{\partial \hat{u}_{\delta t}}{\partial t}+J\left(\check{u}_{\delta t}\right) \check{u}_{\delta t}-\check{p}_{\delta t}\right\rangle .
$$

Let $\phi$ in $C_{c}^{0}(0, T)$ a test function, $\phi \geq 0$. We multiply (74) by $\phi$ and integrate on $(0, T)$ :

$\int_{0}^{T} \chi_{\|\cdot\|_{2} \leq 1}(v) \phi(t) d t \geq \int_{0}^{T} \chi_{\|\cdot\|_{2} \leq 1}\left(\check{u}_{\delta t}\right) \phi(t) d t+\int_{0}^{T}\left\langle v-\check{u}_{\delta t},-\frac{\partial \hat{u}_{\delta t}}{\partial t}+J\left(\check{u}_{\delta t}\right) \check{u}_{\delta t}-\check{p}_{\delta t}\right\rangle \phi(t) d t$

i.e.:

$$
\int_{0}^{T} \chi_{\|\cdot\|_{2} \leq 1}(v) \phi(t) d t \geq \int_{0}^{T} \chi_{\|\cdot\|_{2} \leq 1}\left(\check{u}_{\delta t}\right) \phi(t) d t+\int_{0}^{T} \int_{\Omega}\left(v-\check{u}_{\delta t}\right)\left(-\frac{\partial \hat{u}_{\delta t}}{\partial t}+J\left(\check{u}_{\delta t}\right) \check{u}_{\delta t}-\check{p}_{\delta t}\right) \phi(t) d t d x .
$$

By convexity, we have:

$$
\liminf \int_{0}^{T} \chi_{\|\cdot\|_{2} \leq 1}\left(\check{u}_{\delta t}\right) \phi(t) d t \geq \int_{0}^{T} \chi_{\|\cdot\|_{2} \leq 1}(u) \phi(t) d t .
$$

Now, since $\check{u}_{\delta t} \rightarrow u$ strongly in $L^{2}\left((0, T) ; L^{2}(\Omega)\right), \frac{\partial \hat{u}_{\delta t}}{\partial t} \rightarrow \frac{\partial u}{\partial t}$ in $L^{2}\left((0, T) ; L^{2}(\Omega)\right)$ weak, $J\left(\check{u}_{\delta t}\right) \check{u}_{\delta t} \rightarrow J(u) u$ strongly in $L^{2}\left((0, T) ; L^{2}(\Omega)\right)$, and $\check{p}_{\delta t} \rightarrow p$ strongly in $L^{2}\left((0, T) ; L^{2}(\Omega)\right)$, the second term on the right hand-side of $(76)$ tends to

$$
\int_{0}^{T} \int_{\Omega}(v-u)\left(-\frac{\partial u}{\partial t}+J(u) u-p\right) \phi(t) d t d x .
$$

We thus get: 


$$
\int_{0}^{T} J(v) \phi(t) d t \geq \int_{0}^{T} J(u) \phi(t) d t+\int_{0}^{T} \int_{\Omega}(v-u)\left(-\frac{\partial u}{\partial t}+J(u) u-p\right) \phi(t) d t d x .
$$

This inequality holds for all $\phi \geq 0$, we deduce that for a.e. $t$ in $(0, T)$ :

$$
\chi_{\|\cdot\|_{2} \leq 1}(v) \geq \chi_{\|\cdot\|_{2} \leq 1}(u)+\int_{\Omega}(v-u)\left(-\frac{\partial u}{\partial t}+J(u) u-p\right) d x
$$

i.e.: $-\frac{\partial u}{\partial t}+J(u)-p \in \partial \chi_{\|\cdot\|_{2} \leq 1}(u)$. Hence we deduce that $u$ is a solution of (51) in the distributional sense.

\section{References}

[1] F. Andreu, C. Ballester, V. Caselles, and J. M. Mazón. Minimizing total variation flow. Differential and Integral Equations, 14(3):321-360, 2001.

[2] F. Andreu, V. Caselles, JI Dıaz, and JM Mazón. Some qualitative properties for the total variation flow. Journal of Functional Analysis, 188(2):516-547, 2002.

[3] V. Apidopoulos, J-F. Aujol, and C. Dossal. On a second order differential inclusion modeling the FISTA algorithm. Hal Preprint, (01517708), 2017.

[4] G. Aubert and J-F. Aujol. A variational approach to removing multiplicative noise. SIAM Journal on Applied Mathematics, 68(4):925-946, 2008.

[5] J.-F. Aujol, G. Gilboa, and N. Papadakis. Fundamentals of non-local total variation spectral theory. In Proceedings of the 5th International Conference on Scale Space and Variational Methods in Computer Vision (SSVM'15), pages 66-77, 2015.

[6] A. Belahmidi and A. Chambolle. Time-delay regularization of anisotropic diffusion and image processing. ESSAIM: M2AN, 39(2):231-251, 2005.

[7] G. Bellettini, V. Caselles, and M. Novaga. The total variation flow in $R^{N}$. Journal of Differential Equations, 184(2):475-525, 2002. 
[8] M. Benning, C. Brune, M. Burger, and J. Müller. Higher-order tv methods:enhancement via bregman iteration. J Sci Comput, 54:269$310,2013$.

[9] M. Benning and M. Burger. Ground states and singular vectors of convex variational regularization methods. Meth. Appl. Analysis, 20(4):295-334, 2013.

[10] M. Benning, M. Möller, R.Z. Nossek, M. Burger, D. Cremers, G. Gilboa, and C.-B. Schönlieb. Nonlinear spectral image fusion. arXiv preprint arXiv:1703.08001, 2017.

[11] K. Bredies, K. Kunisch, and T. Pock. Total generalized variation. SIAM J. Imaging Sciences, 3(3):492-526, 2010.

[12] X. Bresson, T. Laurent, D. Uminsky, and J.V. Brecht. Convergence and energy landscape for cheeger cut clustering. In Advances in Neural Information Processing Systems, pages 1385-1393, 2012.

[13] X. Bresson, T. Laurent, D. Uminsky, and J. Von Brecht. Multiclass total variation clustering. In Advances in Neural Information Processing Systems, pages 1421-1429, 2013.

[14] X. Bresson and A.D. Szlam. Total variation, cheeger cuts. In Proceedings of the 27th International Conference on Machine Learning (ICML10), pages 1039-1046, 2010.

[15] X. Bresson, X.-C. Tai, T.F. Chan, and A. Szlam. Multi-class transductive learning based on 1 relaxations of cheeger cut and mumford-shahpotts model. Journal of mathematical imaging and vision, 49(1):191201, 2014.

[16] H. Brezis. Opérateurs maximaux monotones et semi-groupes de contractions dans les espaces de Hilbert. Norht Holland, 1973.

[17] H. Brezis. Analyse fonctionnelle. Théorie et applications. Mathématiques appliquées pour la maitrise. Masson, 1983.

[18] T. Bühler and M. Hein. Spectral clustering based on the graph plaplacian. In Proceedings of the 26th Annual International Conference on Machine Learning, pages 81-88. ACM, 2009.

[19] M. Burger, G. Gilboa, M. Moeller, L. Eckardt, and D. Cremers. Spectral decompositions using one-homogeneous functionals. SIAM Journal on Imaging Sciences, 9(3):1374-1408, 2016. 
[20] M. Burger, G. Gilboa, S. Osher, and J. Xu. Nonlinear inverse scale space methods. Comm. in Math. Sci., 4(1):179-212, 2006.

[21] G. Carlier, M. Comte, and G. Peyré. Approximation of maximal cheeger sets by projection. ESAIM: Mathematical Modelling and Numerical Analysis, 43(1):139-150, 2009.

[22] V. Caselles, A. Chambolle, and M. Novaga. Some remarks on uniqueness and regularity of cheeger sets. Rend. Semin. Mat. Univ. Padova, 123:191-201, 2010.

[23] A. Chambolle and P.L. Lions. Image recovery via total variation minimization and related problems. Numerische Mathematik, 76(3):167$188,1997$.

[24] J.W. Demmel. Applied numerical linear algebra. SIAM, 1997.

[25] J. Duran, M. Moeller, C. Sbert, and D. Cremers. Collaborative total variation: a general framework for vectorial tv models. SIAM Journal on Imaging Sciences, 9(1):116-151, 2016.

[26] G. Gilboa. A total variation spectral framework for scale and texture analysis. SIAM J. Imaging Sciences, 7(4):1937-1961, 2014.

[27] G. Gilboa and S. Osher. Nonlocal operators with applications to image processing. SIAM Multiscale Modeling and Simulation, 7(3):1005-1028, 2008.

[28] M. Hein and T. Bühler. An inverse power method for nonlinear eigenproblems with applications in 1-spectral clustering and sparse pca. In J. D. Lafferty, C. K. I. Williams, J. Shawe-Taylor, R. S. Zemel, and A. Culotta, editors, Advances in Neural Information Processing Systems 23, pages 847-855. Curran Associates, Inc., 2010.

[29] D. Horesh and G. Gilboa. Separation surfaces in the spectral tv domain for texture decomposition. IEEE Transactions on Image Processing, 25(9):4260-4270, 2016.

[30] E. Merkurjev, E. Bae, A.L. Bertozzi, and X.-C. Tai. Global binary optimization on graphs for classification of high-dimensional data. Journal of Mathematical Imaging and Vision, 52(3):414-435, 2015.

[31] E. Merkurjev, T. Kostic, and A.L. Bertozzi. An mbo scheme on graphs for classification and image processing. SIAM Journal on Imaging Sciences, 6(4):1903-1930, 2013. 
[32] B. Merriman, J. K Bence, and S. J. Osher. Diffusion generated motion by mean curvature. In In J. Taylor, editor, Proceedings of the Computational Crystal Growers Workshop, pages 73-83. AMS, 1992.

[33] Y. Meyer. Oscillating patterns in image processing and in some nonlinear evolution equations, March 2001. The 15th Dean Jacquelines B. Lewis Memorial Lectures.

[34] M. Moeller, J. Diebold, G. Gilboa, and D. Cremers. Learning nonlinear spectral filters for color image reconstruction. In Proceedings of the IEEE International Conference on Computer Vision, pages 289-297, 2015.

[35] J. Müller. Advanced image reconstruction and denoising: Bregmanized (higher order) total variation and application in pet, 2013. Ph.D. Thesis, Univ. Münster.

[36] R.Z. Nossek and G. Gilboa. Flows generating nonlinear eigenfunctions. CoRR, abs/1609.08438, 2016.

[37] S. Osher, M. Burger, D. Goldfarb, J. Xu, and W. Yin. An iterative regularization method for total variation based image restoration. SIAM Journal on Multiscale Modeling and Simulation, 4:460-489, 2005.

[38] L. Rudin, S. Osher, and E. Fatemi. Nonlinear total variation based noise removal algorithms. Physica D, 60:259-268, 1992.

[39] Y. Saad. Iterative methods for sparse linear systems. SIAM, 2003.

[40] Marie Foged Schmidt, Martin Benning, and Carola-Bibiane Schönlieb. Inverse scale space decomposition. arXiv preprint arXiv:1612.09203, 2016 .

[41] R. Temam. Navier Stokes equations. Elsevier Science Publishers B.V., 1984.

[42] L. Zeune, G. van Dalum, L. WMM Terstappen, S.A. van Gils, and C. Brune. Multiscale segmentation via bregman distances and nonlinear spectral analysis. SIAM journal on imaging sciences, 10(1):111-146, 2017. 